Abanico Veterinario. Enero-Diciembre 2020; 10:1-12 http://dx.doi.org/10.21929/abavet2020.31

Artículo Original. Recibido: 05/02/2020. Aceptado: 07/11/2020. Publicado: 26/11/2020. Clave:2020-16.

\title{
Propuesta gastronómica con Cavia porcellus
}

\author{
Gastronomic proposal with Cavia porcellus
}

\section{Vargas-Romero Juan $\mathrm{ID}^{*}$, Losada-Custardoy Hermenegildo ${ }^{\mathrm{D}}$, Cortés-Zorrilla JosélD, Alemán-López Viridiana ${ }^{\mathrm{ID}}$, Vieyra-Durán Jorge $\mathrm{e}^{\mathrm{ID}}$, Luna-Rodríguez Lorena ${ }^{\mathrm{ID} * \star}$}

Universidad Autónoma Metropolitana. División de Ciencias Biológicas y de la Salud. Departamento de Biología de la Reproducción. Área de Sistemas de Producción Agropecuarios. Avenida Michoacán y la Purísima, Col. Vicentina, Iztapalapa, C.P. 09340, Ciudad de México, México. *Autor responsable: Juan Vargas-Romero. ${ }^{* *}$ Autor de correspondencia: Lorena Luna-Rodríguez. jmvr@xanum.uam.mx, hrlc@xanum.uam.mx, jocz@xanum.uam.mx, alv@xanum.uam.mx, vdje@xanum.uam.mx,

llunaro@xanum.uam.mx

\section{RESUMEN}

El Cavia porcellus es utilizada como una especie de abasto de carne, es parte de la alimentación y fuente de sustento en algunas regiones del mundo. En México los sistemas de producción de cuyes representan una oportunidad para producir carne para autoconsumo e incluso para venta local. El objetivo de este estudio fue evaluar sensorialmente la propuesta gastronómica a base de carne de $C$. porcellus; la cual estuvo preparada por cuatro guisos (en crema de champiñones, asado, adobado agridulce y en mole amarillito) y se evaluaron sensorialmente por 40 jueces no entrenados mediante una prueba de aceptabilidad con una escala estructurada de satisfacción hedónica, los atributos considerados fueron la apariencia, olor, sabor, terneza y jugosidad. Los datos se analizaron mediante una Clasificación Jerárquica Ascendente y un Análisis de Componentes Principales para construir el modelo cuadrático de mapeo de preferencias externas. Los resultados indicaron que para los consumidores de la clase 2 existe un efecto significativo $\left(R^{2}=0.976, p<0.05\right)$, determinando su aceptación general en común por el cuy en crema de champiñones. Se concluyó que la propuesta gastronómica de los cuatro guisos con carne de cuy fue aceptada, pero el de mayor aceptación fue el cuy en crema de champiñones.

Palabras clave: aceptabilidad, carne, hedónico, prueba sensorial, propiedades organolépticas, no convencional.

\begin{abstract}
Cavia porcellus is used as a kind of meat supply, it is part of the diet and source of sustenance in some regions of the world. In Mexico guinea pig production systems represent an opportunity to produce meat for self-consumption and even for local sale. The objective of this study was to evaluate sensorially the gastronomic proposal based on C. porcellus meat; which consisted of four stews (in mushroom cream, roasted, sweet and sour marinade and in yellow mole) and were tasted by 40 judges not trained by an acceptability test with a structured scale of hedonic satisfaction, the attributes considered were the appearance, smell, taste, tenderness and juiciness. Data were analysed using an Ascendant Hierarchical Classification and Principal Component Analysis to construct the quadratic mapping model of external preferences. The results indicated that for type 2 consumers there is a significant effect $\left(R^{2}=0.976, p<0.05\right)$, determining their general acceptance in common by guinea pig cream mushrooms. It was concluded that the gastronomic proposal of the four guinea pig stews was accepted, but the most widely accepted was the cuy in cream of mushroom soup.
\end{abstract}

Keywords: acceptability, meat, hedonic, sensory test, organoleptic properties, unconventional. 


\section{INTRODUCCIÓN}

El Cavia porcellus (cuy, cuyo, cobayo o cavy doméstico) en la región Andina de América del sur (Perú, Ecuador, Colombia y Bolivia), es considerada como especie de abasto de carne; en esta región la crianza se realiza en sistemas familiares de auto abasto, donde tradicionalmente es utilizada como parte de la alimentación de su población (JuradoGámez et al., 2016; Ayagirwe et al., 2018; Lucas et al., 2018; Dalle-Zotte y Cullere, 2019). De manera semejante en algunos países de África subsahariana (Camerún, República Democrática del Congo y Tanzania), se ha convertido en una fuente de sustento por su importancia social y económica (Matthiesen et al., 2011; Ngoula et al., 2017; Ayagirwe et al., 2018). Otro rasgo a considerar es la composición química de la carne de cuy (humedad 78.2\%, energía $96 \mathrm{kcal}$, proteína 18.8-20.36\%, grasa 2.7-5.1\%, calcio $29 \mathrm{mg}$, fosforo $253 \mathrm{mg}$, hierro $1.9 \mathrm{mg}$, tiamina $0.10 \mathrm{mg}$, riboflavina $0.4 \mathrm{mg}$ y niacina $6 \mathrm{mg}$ (INCAP, 2018), que le atribuyen potencial como fuente alternativa de proteína animal (Avilés et al., 2014; Dalle-Zotte y Cullere, 2019), baja en grasa, en comparación con la carne de otras especies de ganado (Kouakou et al., 2013).

En México, con respecto a la garantía de alcanzar una alimentación adecuada, es importante lo señalado por el Consejo Nacional de Evaluación de la Política de Desarrollo Social (CONEVAL, 2019), que informa que el indicador de acceso a la alimentación describe, que en el $25.5 \%$ de la población mexicana al menos un integrante de la familia modificó, mermó o prescindió de la alimentación por problemas económicos; y que el ingreso de 61.1 millones de personas (48.8 \% de la población), es insuficiente para poder adquirir las canastas alimentaria y no alimentaria.

Xicohtencatl et al. (2013) en Nayarit, México, evaluaron las variables productivas en cuyes y consideraron que los sistemas de producción de cuyes representan una oportunidad de negocio agropecuario familiar, como producción de carne con calidad nutritiva para autoconsumo e incluso de venta local; además, precisaron la necesidad de articular esfuerzos académicos y de gobierno para promocionar esta especie.

Las razones de seguridad alimentaria están claramente establecidas para justificar la propuesta; sin embargo, para este tipo de carne 'no convencional' o 'exóticas' se debe considerar que puede evocar fuertes emociones, actitudes individuales y sociales, controversias e incluso tabúes para la aceptación de la carne de cuy. Debido a la complejidad que esto implica; el objetivo de este estudio fue evaluar sensorialmente la propuesta gastronómica a base de carne de $C$. porcellus. 


\section{MATERIAL Y MÉTODOS}

\section{Origen de los animales}

La procedencia de los $C$. porcellus $(n=15)$ fue de sistemas de producción traspatio, ubicados en la Zona Metropolitana de la Ciudad de México. A continuación, mencionamos algunos aspectos zootécnicos de los cuyes utilizados: la raza fue americana; las crías tuvieron un periodo de lactación de 18 días; después del destete se seleccionaron los machos y se realizó una lotificación homogénea con base al peso, para que se llevara a cabo un buen crecimiento y desarrollo. El tiempo desde el destete al sacrificio fue $5 \pm 0.7$ meses de edad y obtuvieron un peso vivo de $700 \pm 62 \mathrm{~g}$. La crianza de estos animales se realizó con una alimentación mixta [desechos vegetales $(70 \%)$ y concentrado comercial para conejos $(30 \%)$ ], y se garantizó la sanidad e inocuidad en la producción primaria de la carne de cuy.

\section{Descripción de la ración alimenticia}

En el sistema de alimentación se utilizó alimento concentrado comercial (14.5\% de proteína cruda) y se integraban desperdicios orgánicos provenientes de la CEDA desechos vegetales (no convencionales). En el cuadro 1 se observan los valores nutricionales de los alimentos no convencionales utilizados en este estudio, que fueron evaluados y reportados previamente por nuestro equipo de trabajo (Almaráz et al., 2012). Con estos datos, se calculó los requerimientos nutricionales recomendados por Xicohtencatl et al. (2013).

Cuadro 1. Análisis químico de los ingredientes no convencionales (desechos vegetales)

\begin{tabular}{lccccc}
\hline & Col & Coliflor & Hojas de elote & Lechuga & Concentrado comercial \\
\hline Humedad, \% & 88.5 & 89.9 & 81.29 & 94.30 & 12 \\
MS, \% & 11.5 & 10.1 & 18.30 & 5.75 & 88 \\
Cenizas, \% & 16.5 & 14.4 & 3.45 & 23.20 & 10 \\
PC, \% & 10.7 & 18.3 & 3.70 & 17.20 & 14.50 \\
FDN, \% & 18.5 & 22.9 & 69.90 & 26.20 & 18 \\
FDA, \% & 13.6 & 16.5 & 32.20 & 19.70 & 13 \\
Hem, \% & 4.9 & 6.4 & 37.70 & 6.40 & -- \\
Lignina, \% & 2.7 & 2.6 & 1.40 & 9.60 & -- \\
\hline
\end{tabular}

\section{Obtención de la canal de cuy}

Se realizó el sacrificio siguiendo los protocolos de bienestar animal, la insensibilización fue mediante la exposición con $\mathrm{CO}_{2}$ al $90 \%$, por 17 min (AVMA, 2020), finalizado este tiempo se efectuó un corte transversal en la vena yugular para desangrar. Posteriormente las canales se colocaron en posición caudo-craneal para proceder al faenado (desollado y eviscerado) (Mota-Rojas et al., 2012) y después las canales (417 $\mathrm{g} \pm 60$ ) se conservaron a $4^{\circ} \mathrm{C}$ durante $24 \mathrm{~h}$ (Rubio et al., 2014); luego cada canal fue separada acorde al protocolo de Sánchez-Macías et al. (2016), separando e identificando la porción de la pierna larga. 
Finalmente, las muestras se colocaron a $-20^{\circ} \mathrm{C}$ (Rodbotten, 2004), para su posterior utilización en las pruebas sensoriales.

\section{Preparación de la muestra}

Se siguió un protocolo de descongelación lenta (periodo de $24 \mathrm{~h}$ ), hasta obtener una temperatura interna de $5^{\circ} \mathrm{C}$ en las muestras (Font, 2006), y luego se retiró el tejido conectivo y la grasa de cobertura.

\section{Características de los consumidores y prueba sensorial}

Fue presentada la propuesta gastronómica a los jueces-consumidores, en un contexto de consumo lo más cercano a la realidad, el criterio básico fue simular las condiciones de 'Experiencia Gastronómica' de la manera más fidedigna posible. A pesar de lo anterior el área donde se realizó la evaluación permitió tener el control, como la iluminación (luz blanca), temperatura $\left(25^{\circ} \mathrm{C}\right)$, ruido y olores extraños. El horario de la evaluación fue a las 11:00 $\mathrm{h}$ (después de desayunar y antes de comer.

Esta propuesta gastronómica estuvo integrada por cuatro guisos con carne de cuy (en crema de champiñones, asado, adobado agridulce y en mole amarillito). Los guisos fueron elaborados con ingredientes de uso común en México (cuadro 1), y se utilizaron 3 sistemas de cocción; dos de estos analizados en un estudio preliminar por este grupo de investigación (Luna et al., 2017); para cada una de las técnicas se obtuvo una temperatura interna de $75^{\circ} \mathrm{C}$ (en el centro geométrico) en la carne.

Cuadro 2. Descripción de los guisos con ingredientes de uso común en México

\begin{tabular}{|c|c|c|}
\hline Guiso con carne de cuy & Método culinario & Ingredientes \\
\hline $\begin{array}{l}\text { Crema } \\
\text { champiñones }^{a, b, f}\end{array}$ & Frito & $\begin{array}{l}\text { Champiñones blancos, hongos portobello, hongos } \\
\text { girgolas (setas), crema para batir, fondo de ave, } \\
\text { mantequilla clarificada y nuez caramelizada. }\end{array}$ \\
\hline Asado ${ }^{a, b, c, d, e, f}$ & Parrilla & Chile guajillo, chile de árbol y vinagre de manzana. \\
\hline Adobado agridulce ${ }^{a, b, c, d, e, f}$ & Inmersión & $\begin{array}{l}\text { Chile ancho, chile guajillo, vinagre blanco, orégano, } \\
\text { duraznos en almíbar y sal. }\end{array}$ \\
\hline Mole amarillito ${ }^{\mathrm{a}, \mathrm{b}, \mathrm{c}, \mathrm{d}, \mathrm{f}}$ & Inmersión & $\begin{array}{l}\text { Chile chilhuacle amarillo, tomate verde, jitomate, hoja } \\
\text { santa, ajonjolí tostado, aceite, caldo, masa de maíz, } \\
\text { calabacita italiana en juliana y chicharos. }\end{array}$ \\
\hline
\end{tabular}

Ingredientes para más de un guiso: ajo ${ }^{a}$, cebolla ${ }^{b}$, pimienta $^{c}$, clavo de olor ${ }^{d}$, comino $^{e}$, sal $^{f}$

Fueron reclutados 40 consumidores de ambos sexos, con un rango de edad de 20 a 52 años, los cuales indicaron que eran consumidores habituales de carne; además les fue informado el tipo de carne a evaluar. Para la prueba de aceptación a cada consumidor se le presentaron los cuatro guisos (a $55^{\circ} \mathrm{C}$ ) emplatados (en 4 tiempos y en platos lisos de color blanco) y se les proporcionó agua como agente limpiador del gusto, la cual utilizaron antes de la evaluación de cada guiso (Peachey, 2002). 
Se usó la metodología afectiva (González et al., 2017) con una escala estructurada de satisfacción hedónica de 7 puntos (me desagrada mucho: -3; me desagrada medianamente: -2; me desagrada: -1 ; ni me desagrada ni me agrada: 0; me agrada: 1; me agrada medianamente: 2 y me agrada mucho: 3 ). Las variables sensoriales evaluadas fueron terneza, jugosidad, sabor y olor.

\section{Análisis estadísticos}

Se utilizó el software XLSTAT-Sensory versión 2015.6.01.25740 (Copyright Addinsoft 1995-2016). Para el estudio de aceptación, se realizó una agrupación de consumidores que generó las clases para la variable de apreciación global asociada con los cuatro atributos evaluados en la prueba sensorial; es decir, se utilizó una Clasificación Jerárquica Ascendente (CJA) y un Análisis de Componentes Principales (ACP) de los atributos sensoriales para construir el modelo cuadrático de mapeo de preferencias externas (MPE) (González et al., 2017).

\section{RESULTADOS Y DISCUSIÓN}

\section{Estudio de la aceptación de la carne de Cuy}

El análisis sensorial puede ser una buena herramienta para desarrollar nuevos productos en un restaurante, al simular las condiciones en las que se puede ofrecer esta carne; la propuesta permitió evaluar su percepción en una situación realista. En el espacio sensorial (figura 1), los atributos sensoriales que se asocian al primer componente principal (F1) son olor, sabor y terneza y el atributo asociado al segundo componente (F2) fue la jugosidad y representó al $90.99 \%$ de la variabilidad de los datos de aceptación. Se observó que los consumidores tienen percepciones diferentes en los atributos; lo anterior, debido a la técnica culinaria y a los ingredientes utilizados.

La propuesta de mayor aceptación fue el cuy preparado en crema de champiñones, ubicándose en los valores positivos para ambos componentes principales y en particular en la preferencia asociada al atributo de sabor al presentarse más fuerte, ya que se aleja de la dirección indicada por el vector. El cuy asado obtuvo valores positivos para olor sabor y terneza, pero negativos para jugosidad; por lo cual, esta preparación culinaria fue de menor aceptación.

El mapa externo de preferencia (figura 2 y 3 ) indica que para los consumidores de la clase 2 , existe un efecto significativo $\left(R^{2}=0.976, p<0.05\right)$; ya que con dicho modelo se explicó las preferencias de este grupo de consumidores, determinando su aceptación general en común por el cuy en crema de champiñones. La clase 1 y clase 3 tienen una $R^{2}=0.968$ y $R^{2}=0.893(p<0.05)$ lo que determina su aceptación general en común por el cuy en mole amarillito y en crema de champiñones respectivamente.

Para los consumidores pertenecientes a la clase $4\left(R^{2}=0.081\right)$, el modelo aplicado en la presente investigación no pudo explicar las preferencias. La clase 2 y clase 3 , muestran preferencias similares para el cuy en crema de champiñones, ya que este guiso es el de 
mayor preferencia en ambas clases. El porcentaje de los consumidores satisfechos (figura 3), fue del $60-80 \%$ para los guisos de cuy en crema de champiñones, en mole amarillito y adobado agridulce y de $40-60 \%$ para el cuy asado.

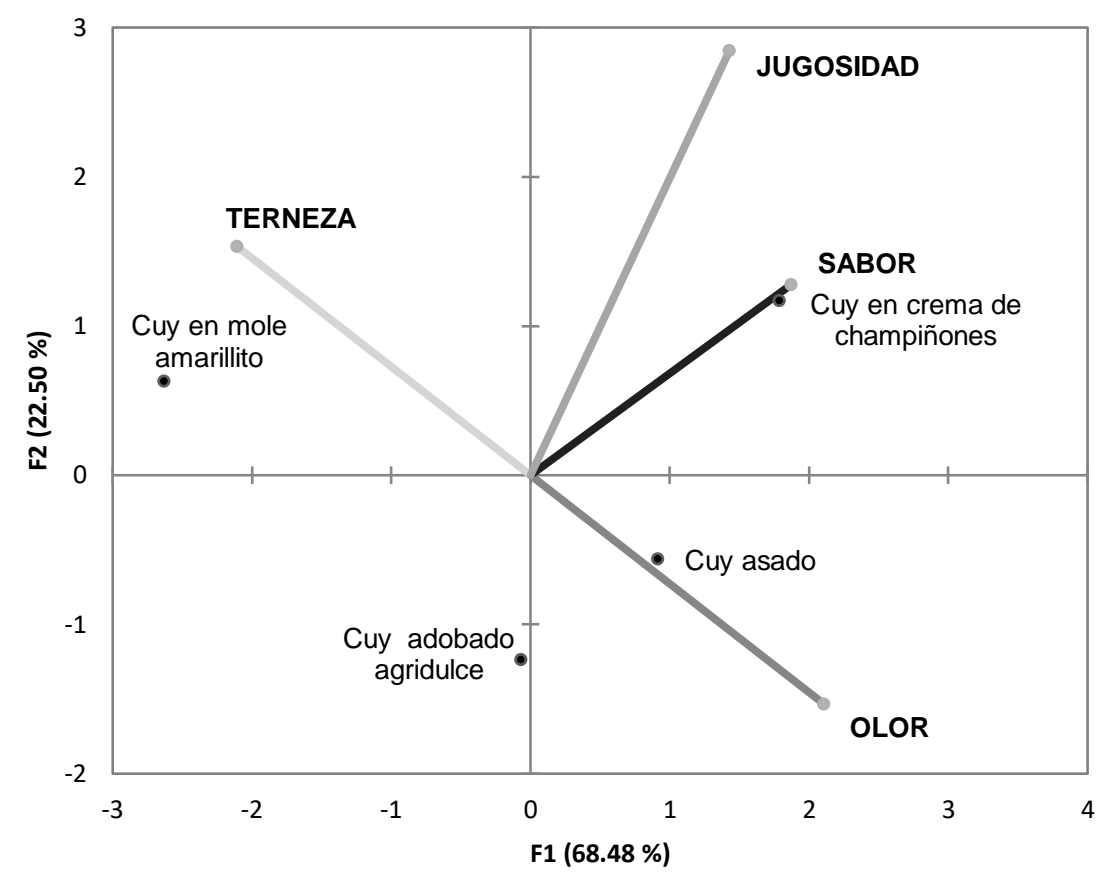

Figura 1. Espacio sensorial de la propuesta gastronómica con carne de Cavia porcellus. Los atributos sensoriales fueron calificados mediante una escala hedónica de aceptación de 7 puntos.

Barham et al. (2010) indicaron que el juicio de la calidad de un guiso es un asunto personal, como la medida en que se disfruta o no una comida en particular y que se deben de cumplir una serie de condiciones (sabor, textura, jugosidad y olor), antes de que la comida sea realmente agradable. En el presente trabajo a pesar del uso de diferentes técnicas culinarias para la elaboración de estos guisos las puntuaciones sensoriales de la escala hedónica estructurada (bipolar) los sitúan en valores positivos, lo que indica que el consumidor aceptó este tipo de carne en distintas presentaciones.

Al dilucidar las condiciones por las cuales los guisos fueron descritos por este grupo de individuos en estudio como agradables, cabe mencionar la forma en que pudieron cumplir con las condiciones para ello; y fue a través de la producción de materias primas (que para todos los guisos fue la misma), en el proceso de cocción (distintas técnicas culinarias) y en la forma en que se presentan los alimentos (ingredientes variables);por supuesto, con la preparación de los guisos (ingredientes y su combinación). Además del proceso de cocción realizada de una manera apropiada, puede llevar al desarrollo de calidad de un plato de comida. En algunos guisos se involucraron procesos que 
desarrollaron el sabor, como se observa en el cuy en crema de champiñones, que obtienen calificaciones positivas para sabor y en otros este puede afectar las características organolépticas, como sucedió con el asado que fue el menos aceptado y obtuvo calificaciones negativas en jugosidad.

Natividad et al. (2010) y Flores-Mancheno et al. (2017) elaboraron embutidos con carne de cuy reportando buenas características organolépticas y Apráez (2011), obtuvo la aceptación para el chorizo de cuy en un $21,67 \%$ con diferencias significativas para la categoría 'me gusta muchísimo', para el chorizo de cuy. También se han realizado otras evaluaciones sensoriales para la carne de $C$. porcellus, en distintas presentaciones y la carne de cuy ahumada fue la de mayor aceptación, seguida de la carne de cuy empanizada y en croquetas (Argote et al., 2009).

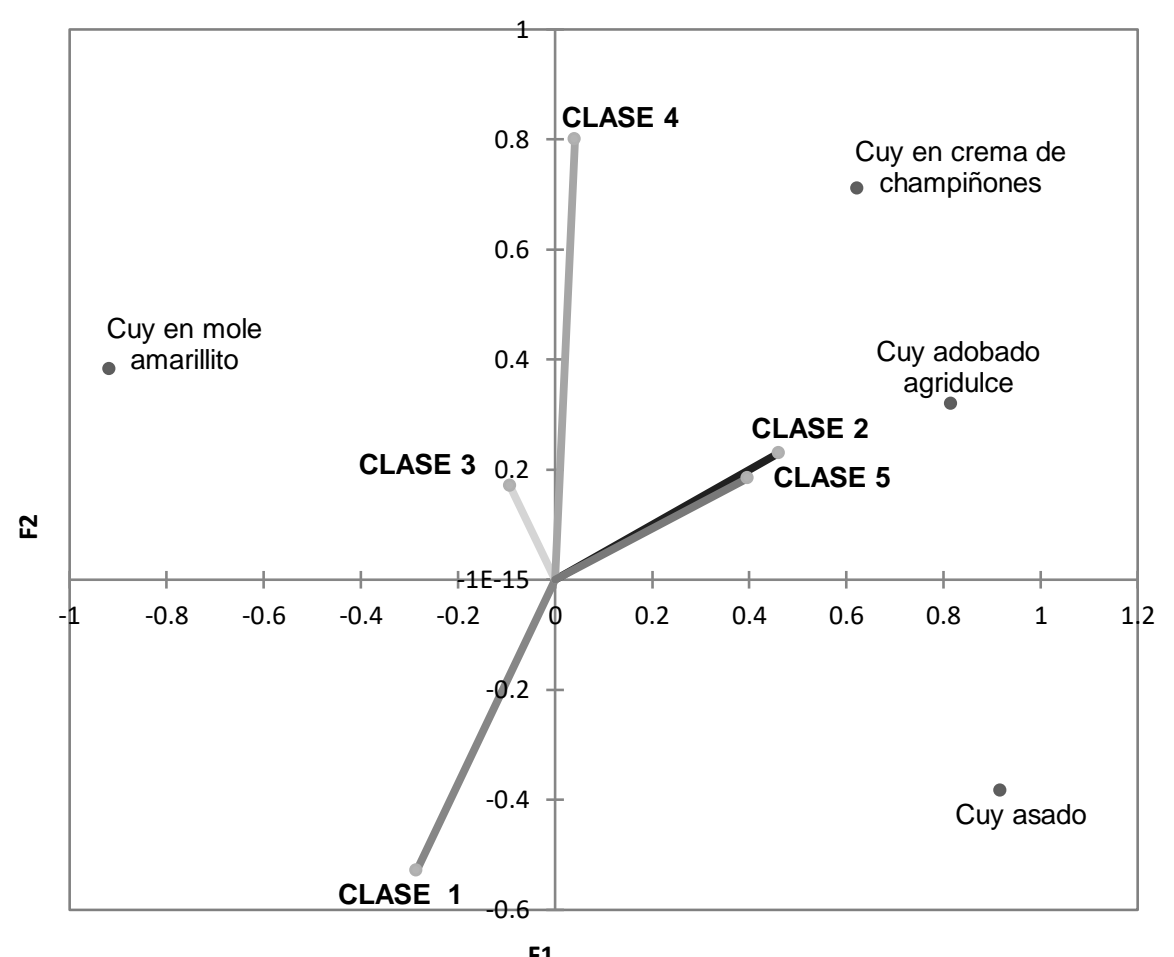

Figura 2. Mapa externo de preferencia modelo vectorial para la aceptación general de carne de Cavia porcellus, calificada mediante una escala hedónica de aceptación de 7 puntos.

También, Argote et al., (2009) mediante un modelo cuantitativo y muestreo probabilístico aleatorio, realizó la colección de la información sobre la preferencia de la carne de cuy en Colombia (consumo carne de res por tradición), encontrando que el $96 \%$ de la población encuestada ha consumido carne de cuy, y el resto (4\%) no lo hace porque no le gusta su sabor y apariencia. Por tanto, la incorporación de este tipo de alimento es viable, ya que los resultados obtenidos en la escala hedónica para los diferentes platillos son calificados como: me agrada, me agrada medianamente y me agrada mucho. 
De manera similar al presente estudio, Yaguache (2013) propuso alternativas culinarias utilizando la carne de cuy y las evaluó sensorialmente mediante una escala hedónica; los platos preparados fueron bien aceptados. El $45 \%, 46 \%, 54 \%$ y $44 \%$ de los consumidores indicaron que les gustó mucho las brochetas de cuy, chicharrón de cuy, enrollado de cuy y el cuy al vino, respectivamente. Por lo cual, se reportó que la carne de cuy gusta a todos los paladares en diferentes formas de prepararla.

El cuy está ganando importancia como fuente de carne exótica, y por consiguiente su importancia como animal productor de carne está aumentando; pero aún falta investigar sobre sus rasgos de calidad de la carne y es necesario ser explorada con mayor profundidad.

Sin embargo, en posteriores estudios es importante la utilización de la especie $C$. porcellus adaptada en México y no considerar trasladar animales nativos de Sudamérica, ya que según lo sugerido por la FAO (2010), «la diversidad biológica posee un valor intrínseco y debe conservarse por sí misma al máximo nivel posible».

La adquisición de esta carne puede ser viable, por lo señalado por Xicohtencatl et al. (2013) quienes indican un costo de producción de un cuy al destete de $\$ 30.14$ pesos $\mathrm{MN}$ e infieren que los sistemas de producción de cuy pueden tener ingresos de la venta de cuyes para mascota y también de la producción de carne para consumo local.

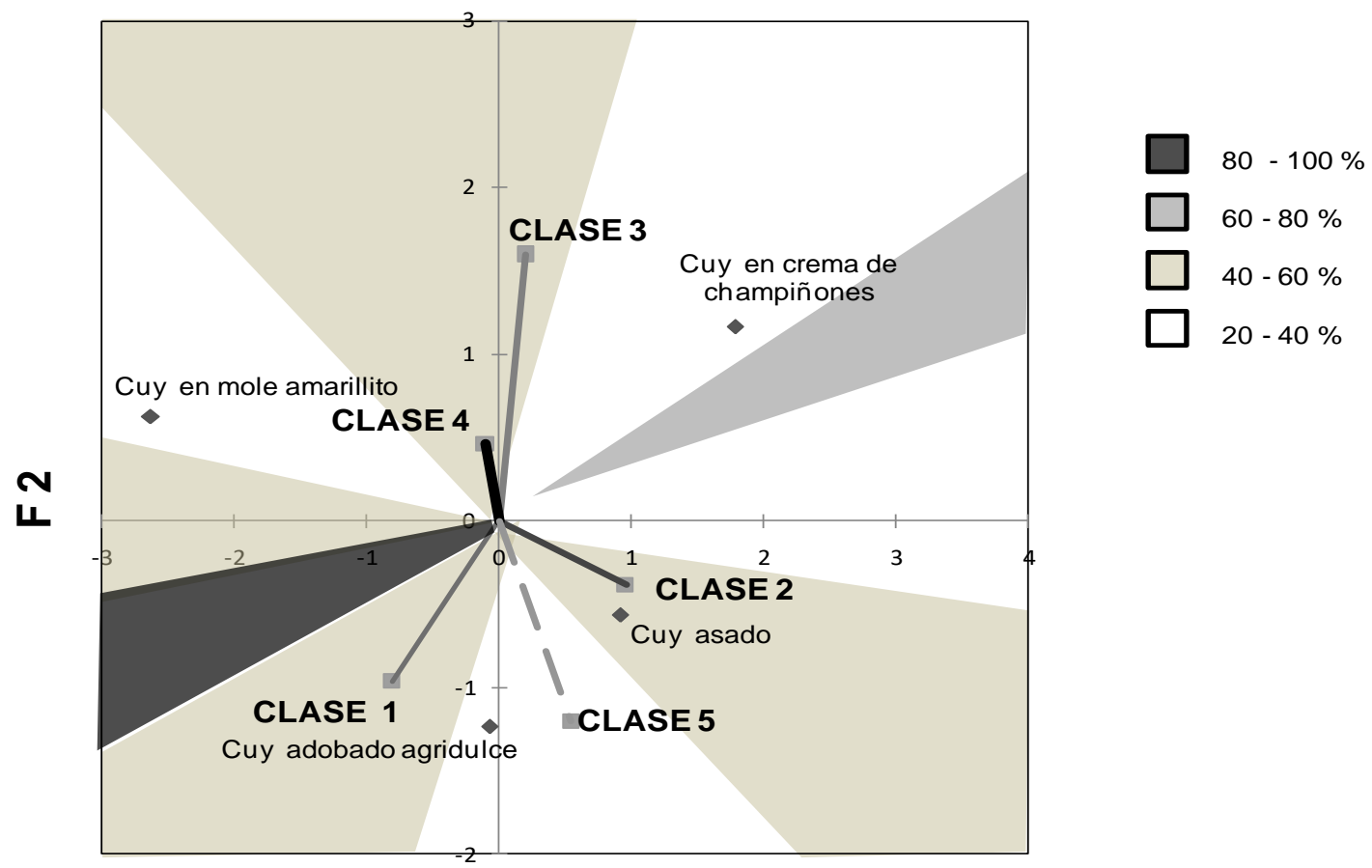

F 1

Figura 3. Mapa de preferencias y gráfico de contorno de los porcentajes de satisfacción utilizando un modelo vectorial para la aceptación general para la carne de Cavia porcellus, calificada mediante una escala hedónica de aceptación de 7 puntos. 


\section{CONCLUSIONES}

La sustitución de carne de animales de abasto tradicionales por carne de cuy, no afecta los atributos sensoriales y es aceptada por los consumidores. Este tipo de carne fue recibida de manera positiva, ya que los jueces al valorar la propuesta gastronómica utilizando la carne de cuy tuvieron un alto porcentaje de satisfacción. Por lo tanto, la carne de $C$. porcellus puede posicionarse como una carne atractiva para el consumidor. Además, al realizar la evaluación lo más apegado a una experiencia de un servicio de comida, se obtuvo información para considerar que la carne de cuy es una alternativa como fuente alimenticia no tradicional y que se puede incorporar al mercado gastronómico.

\section{LITERATURA CITADA}

ALMARAZ I, Losada H, Cortés J, Vargas J, Miranda L, Sánchez J. 2012. Producción de gas in Vitro de desechos de verduras usados para alimentar vacas lecheras. Livestock Research for Rural Development. 24(8). Documento 132. ISSN:0121-3784. http://www.Irrd.org//rrd24/8/ alma24132.htm

APRÁEZ GJE, Fernández PL, Hernández GA. 2011. Evaluación de diferentes formas de presentación de la carne de cuy (Cavia porcellus). Veterinaria y Zootecnia. 5(2):24-29. ISSN:2011-5415. http://vetzootec.ucaldas.edu.co/downloads/v5n2a02.pdf

ARGOTE VF, Villada CHS, Argote H. 2009. Investigación de mercado sobre el grado de aceptación de la carne de cuy (Cavia porcellus) en presentaciones de ahumado, croquetas y apanado en la ciudad de Pasto. Biotecnología en el Sector Agropecuario y Agroindustrial. $7(1): 55-66$.

ISSN:1692-3561. https://revistas.unicauca.edu.co/index.php/biotecnologia/article/view/701/329

AVILÉS D, Landi V, Delgado J, Martínez A. 2014. El pueblo ecuatoriano y su relación con el cuy. Actas Iberoamericanas de Conservación Animal. 4:38-40. ISSN:2253-9727. http://www.uco.es/conbiand/aica/templatemo_110_lin_photo/articulos/2014/Trabajo009_ AICA2014.pdf

AVMA (American Veterinary Medical Association). 2020. Guidelines for the euthanasia of animals: 2020 edition. ISBN:978-1-882691-54-8. https://www.avma.org/sites/default/files/2020-01/2020_Euthanasia_Final_1-15-20.pdf

AYAGIRWE RBB, Meutchieye F, Manjeli Y,Maass BL. 2018. Production systems, phenotypic and genetic diversity, and performance of cavy reared in sub-Saharan Africa: a review. Livestock Researchfor Rural Development. 30(6):1-18. ISSN:0121-3784. http://www.Irrd.org/lrrd30/6/ayagi30105.html 
BARHAM P, Leif H, Skibsted, Wender LP Bredie, Michael Bom Frost, Per Moller, Jens Risbo, Pia Snitkjær, Louise Morch Mortensen. 2010. Molecular Gastronomy: A New Emerging Scientific Discipline. Chem Reviews. 110(4):2313-2365. https://doi.org/10.1021/cr900105w

CONEVAL (Consejo Nacional de Evaluación de la Política de Desarrollo Rural). 2019. Diez años de medición de pobreza multidimensional en México: avances y desafíos en política social Medición de la pobreza serie 2008-2018. https://www.coneval.org.mx/Medicion/MP/Documents/Pobreza_18/Pobreza_2018_CON EVAL.pdf

DALLE-ZOTTE A, Cullere M. 2019. Carcass traits and meat quality of rabbit, hare, guinea pig and capibara. In JM Lorenzo, P Munekataa, FJ Barba, F Toldrá (eds), More tan beef, pork and chicken-The production, processing, and quality traits of other sources of meat for Human diet. Springer Nature Switzerland AG. ISBN.978-3-030-05483-0. https://doi.org/10.1007/978-3-030-05484-7_7

FAO (Food and Agriculture Organization). 2010. La situación de los recursos zoogenéticos mundiales para la alimentación y la agricultura, ed. Rischkowsky B, Pilling D. http://www.fao.org/3/a-a1250s.pdf

FLORES-MANCHENO CI, Duarte C, Salgado-Tello PI. 2017. Characterization of the guinea pig (Cavia porcellus) meat for fermented sausage preparation. Revista Ciencia y Agricultura. $14(1): 39-\quad 45$.

ISSN:0122-8420.

https://dialnet.unirioja.es/servlet/articulo?codigo=5971205

FONT MR, Guerrero L, Sañudo C, Campo MM, Olleta JL, Oliver MA, Cañeque V, Álvarez I, Díaz MT, Branscheid W, Wicke M, Nute GR and Montossi F. 2006. Acceptability of lamb meat from different producing systems and ageing time to German, Spanish and British consumers. Meat Science. 72:545-554. ISSN.0309-1740. https://doi.org/10.1016/j.meatsci.2005.09.002

GONZÁLEZ GL, Luna RL, Carrillo LLM, Alarcón RAD, García Gl, Reyes VR. 2017. Ultrasound as an Alternative to Conventional Marination: Acceptability and Mass Transfer. $\begin{array}{llll}\text { Journal of Food Quality. 2017(ID 8675720):8. ISSN:1745-4557 } & \end{array}$ https://doi.org/10.1155/2017/8675720

INCAP (Instituto de Nutrición de Centro América y Panamá). 2018. Tabla de Composición de Alimentos de Centroamérica, /INCAP/Menchú, MT ed; Méndez, H. ed. Guatemala: 
INCAP/OPS, segunda edición. Pp.366. ISBN:978-9929-701-17-5. https://issuu.com/incap1/docs/tca_incap_3ra_edici_n_segunda_reimpresi_n_2020

JURADO-GÁMEZ H, Cabrera-Lara E, Salazar J. 2016. Comparación de dos tipos de sacrificio y diferentes tiempos de maduración sobre variables fisicoquímicas y microbiológicas de la carne de cuy (Cavia porcellus). Revista Médica Veterinaria y Zootecnia. 63(3):201-217. ISSN:0120-

2952.

http://dx.doi.org/10.15446/rfmvZ.v63n3.62741

KOUAKOU NG DV, Grongnet JF, Assidjo EN, Thys E, Marnet PG, Catheline D, Legrand $P$ y Kouba M. 2013. Effect of a supplementation of Euphorbia heterophylla on nutritional meat quality of Guinea pig (Cavia porcellus L.). Meat Science. 93(4): 821-826. ISSN:03091740. https://doi.org/10.1016/j.meatsci.2012.11.036

LUCAS JR, Balcázar S, Tirado O, Rodríguez A. 2018. El pH de la carne de cobayo (Cavia porcellus) para consumo humano en los andes centrales del Perú. Revista Veterinaria. 29(1):65-67. ISSN:1669-6840. http://dx.doi.org/10.30972/vet.2912793

LUNA RL, Losada CHR, Sánchez CR, Cortés ZJ, Vieyra JE, Alemán LV, Rodríguez FR, Vargas RJM. 2017. Cooking methods of Cavia porcellus meat. NACAMEH. 11(2): 58-65. ISSN:2007-0373.

http://nacameh.cbsuami.org/volumenes/v11n2/Nacameh_v11n2p58_LunaRdz-ycol.pdf

MATTHIESEN T, Nyamete F, Msuya JM, Maass BL. 2011. Importance of Guinea Pig Husbandry for the Livelihood of Rural People in Tanzania: A Case Study in Iringa Region. In "Development on the margin" Tropentag, Bonn, DE. 1 p. https://cgspace.cgiar.org/bitstream/handle/10568/65629/Matthiesen_IIDdf2DY.pdf?sequ ence $=1$

MOTA-ROJAS D, Trujillo-Ortega ME, Becerril-Herrera M, Roldán-Santiago P. 2012. Efecto del método de sacrificio sobre variables críticas sanguíneas y consecuencias sobre la bioquímica de la carne de cuyo (Cavia porcellus). Revista Cientifica FCV-LUZ. 12(1):51-58. ISSN:0798-2259.

http://www.fmvz.unam.mx/fmvz/secretarias/general/articulos/50.pdf

NATIVIDAD BAD, Rojas PM, Matos RAM, Muñoz GS. 2010. Uso de la carne de cuy (Cavia cutleri) en la obtención de cuatro tipos de embutidos. Investigación Valdizana. 4(1):1-8. ISSN: 1995-445X. http://revistas.unheval.edu.pe/index.php/riv/issue/view/8/7

NGOULA F, Guemdjo TM, Kenfack A, Tadondjou CDA, Nouboudem S, Ngoumtsop H Tsafacka B, Teguia A, Kamtchouing P, Galeotti M and Tchoumboue J. 2017. Effects of 
heat stress on some reproductive parameters of male cavia (Cavia porcellus) and mitigation strategies using guava (Psidium guajava) leaves essential oil. Journal of Thermal Biology. (64):67-72.

ISSN:0306-4565.

https://doi.org/10.1016/j.jtherbio.2017.01.001

PEACHEY BM, Purchas RW, Duizer LM. 2002. Relationships between sensory and objective measures of meat tenderness of beef $\mathrm{m}$. longissimus thoracis from bulls and steers. Meat Science. 60(3):211-218. ISSN:0309-1740. https://doi.org/10.1016/S03091740(01)00123-1

RODBOTTEN M, Kubberod E, Lea P, Ueland O. 2004. A sensory map of the meat universe. Sensory profile of meat from 15 species. Meat Science. (68):137-144. ISSN:0309-1740. https://doi.org/10.1016/j.meatsci.2004.02.016

RUBIO LMS, Pérez BEV, Méndez MRD, Chávez GA, Delgado SEJ. 2014. La estrategia de congelación, descongelación y maduración mejora la suavidad de los músculos del cuarto delantero de toretes sin castrar de diferentes fenotipos. Veterinaria México. 1(1):19. ISSN:0301-5092. https://www.medigraphic.com/pdfs/vetmex/vm-2014/vm141d.pdf

SÁNCHEZ-MACÍAS D, Castro N, Rivero M, Argüello A, Morales-de la Nuez A. 2016. Proposal for standard methods and procedure for guinea pig carcass evaluation, jointing and tissue separation. Journal of Applied Animal Research. 44: 65-70. ISSN:0974-1844. https://doi.org/10.1080/09712119.2015.1006234

YAGUACHE AAB. 2013. Alternativas culinarias utilizando la carne de cuy para la empresa señor cuy Riobamba, Escuela Superior Politécnica de Chimborazo. Facultad de Salud Pública Escuela de Gastronomía. Licenciatura en Gestión Gastronómica. Ecuador. http://dspace.espoch.edu.ec/bitstream/123456789/9619/1/84T00210.pdf

XICOHTENCATL-SÁNCHEZ PG, Barrera-Zúñiga S, Orozco-Orozco T, Torres-Sandoval SFM, Monsiváis-Isiordia R. 2013. Parámetros productivos de cuyes (Cavia Porcellus) del nacimiento al sacrificio en Nayarit, México. Abanico Veterinario. 3(1):36-43. ISSN:20074204. https://www.medigraphic.com/pdfs/abanico/av-2013/av131e.pdf 